\title{
Acute Respiratory Distress after Knee Arthroplasty Due to Undiagnosed Atrial Myxoma
}

\author{
Zeest Khan ${ }^{1}$, Bo Yang ${ }^{2}$, Hari Mallidi ${ }^{2}$, Pieter van der Starre ${ }^{1}$ \\ ${ }^{1}$ Departments of Anesthesia, School of Medicine, Stanford University, Stanford, USA; ${ }^{2}$ Cardiothoracic Surgery, School of Medicine, \\ Stanford University, Stanford, USA. \\ Email: pieterva@stanford.edu
}

Received April $5^{\text {th }}, 2012$; revised May $13^{\text {th }}, 2012$; accepted June $10^{\text {th }}, 2012$

\begin{abstract}
Acute postoperative respiratory distress may be caused by pneumonia, pulmonary embolism, heart failure, pneumothorax or atelectasis. We present $\mathrm{s}$ case report of a 78-year-old female patient who developed acute respiratory distress one day following knee arthroscopy. Because of the suspicion of pulmonary embolism, a computed tomographic angiography was made, showing the presence of an unexpected large left atrial mass, suggestive for a myxoma. She underwent successful resection of a $10 \times 5 \mathrm{~cm}$ myxoma one week later. The report discusses the clinical manifestations of an atrial myxoma and its potentially fast growth. Bedside echocardiography should be considered the preferred diagnostic modality in case of acute postoperative respiratory distress.
\end{abstract}

Keywords: Non-Cardiac Surgery; Acute Respiratory Distress; Left Atrial Myxoma; Echocardiography; Cardiac Surgery

\section{Introduction}

Acute postoperative respiratory distress following noncardiac surgery can be a serious adverse event, and is most often caused by pneumonia, pulmonary embolus, pneumothorax, atelectasis and heart failure [1]. In this brief report we present a case of acute postoperative pulmonary edema due to an unsuspected, previously asymptomatic, large left atrial myxoma.

\section{Case}

A 78-year-old woman with a history of hypertension, osteoarthritis, and a recent diagnosis of asthma underwent total knee arthroscopy under general anesthesia. Cardiac history was otherwise negative. On postoperative day one, she developed acute shortness of breath, tachypnea, and cough. Workup included a chest X-ray demonstrating bilateral pleural effusions, and a normal ECG. In concern for pulmonary embolism, computer tomographic angiography (CTA) was performed, which was negative for pulmonary embolism, but significant for a large left atrial mass. Subsequent transthoracic echocardiography demonstrated a mobile mass measuring $6 \times 3 \mathrm{~cm}$, with an attachment to the interatrial septum, consistent with left atrial myxoma.

Of note, the patient underwent a CT chest eight months earlier for symptoms of fatigue and intermittent cough.
No significant abnormalities were observed, and she was subsequently treated for asthma.

On postoperative day seven, after successful treatment of pulmonary edema, the patient underwent a left atrial myxoma resection on cardiopulmonary bypass. Intraoperative transesophageal echocardiography (TEE) demonstrated a large mobile left atrial mass, with a septal attachment near the mitral valve, causing mitral valve obstruction and moderate to severe mitral regurgitation (Figure 1). The left atrial mass was resected via a right atrial transseptal approach, measured $10 \times 5 \mathrm{~cm}$ (Figure 2), and was diagnosed on pathologic specimen as an atrial myxoma. TEE after mass resection demonstrated that all signs of mitral stenosis were alleviated, with a residual mild mitral regurgitation.

Apart from developing episodes of hemodynamically stable atrial fibrillation, the patient's postoperative course was uneventful, and she was discharged on postoperative day 7.

\section{Discussion}

Atrial myxoma is the most common type of primary cardiac tumors, with an annual incidence of 0.5 per million [2]. They demonstrate a female predominance, and $75 \%$ originate from the left atrial fossa ovalis [3]. The most common symptoms are the clinical manifestations of mitral stenosis, and include cough, dyspnea, pulmonary 


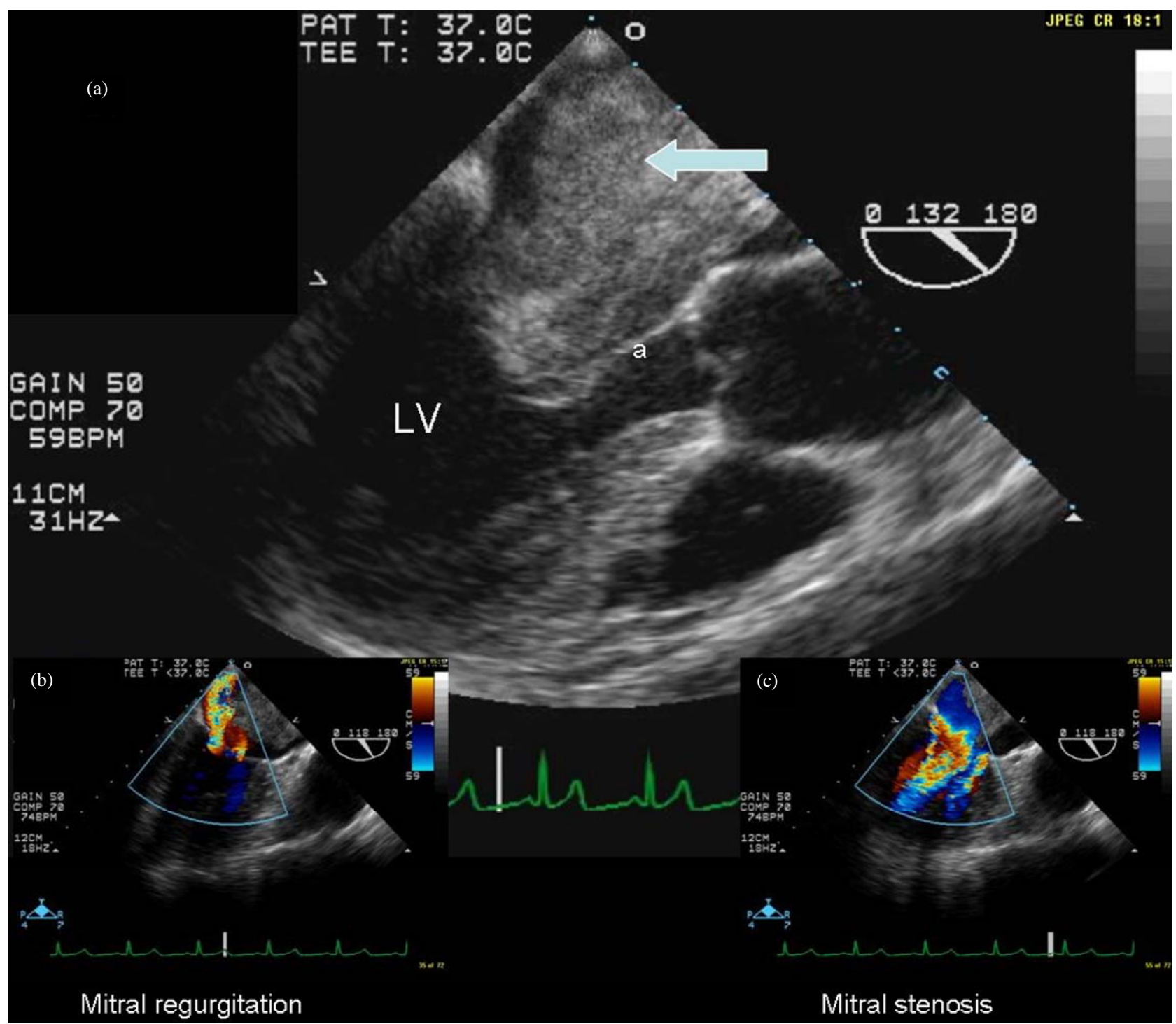

Figure 1. Intraoperative Transesophageal Echocardiography (long-axis view), showing: (a) Large left atrial myxoma (arrow), obstructing inflow through the mitral valve annulus during diastole $(a=$ anterior leaflet of mitral valve). $L V=$ left ventricle; (b) Myxoma causing mitral regurgitation; (c) Myxoma causing mitral stenosis.

edema, and pulmonary hypertension. Left atrial enlargement may lead to atrial arrhythmias, thrombus formation, and embolism. More systemic symptoms like fever, arthralgia, fatigue and weight-loss are described as well. As many as $4 \%$ of patients remain asymptomatic [4]. Atrial myxomas may grow quite rapidly, with a reported growth rates of average $0.49 \mathrm{~cm} /$ month [5]. Our patient had no detectable mass by CT only eight months earlier.

Myxomas cause mitral stenosis by obstructing the mitral valve during diastole. While the pathophysiology is distinct from mitral stenosis caused by rheumatic disease, the mitral stenosis may be as severe, and the clinical consequences are the same. Furthermore, acute obstruction of the mitral valve caused by a very large myxoma may lead to cardiovascular collapse that cannot be re- lieved without surgical intervention.

Mitral regurgitation often coexists, and may result from damage to the mitral valve leaflets by the mass, most often the anterior leaflet, or from annular dilation. This may require annuloplasty after myxoma resection [6].

The patient's acute respiratory distress is probably due to acute obstruction of the mitral valve by the myxoma. As with mitral stenosis from rheumatic disease, the presence of a left atrial myxoma requires appropriate volume status and systemic vascular resistance. An alteration in these parameters may have lead to pulmonary overload. It is fortunate that our patient received a general anesthetic for her knee surgery, as a spinal anesthetic-induced reduction in preload and systemic vascular resistance could have caused immediate circulatory col- 


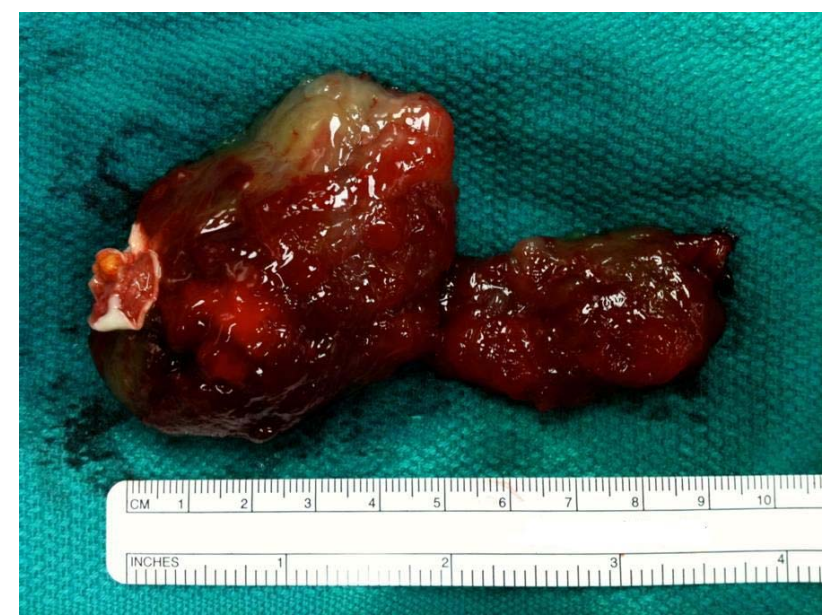

Figure 2. Specimen: left atrial myxoma with atrial septal attachment, measuring $6 \times 3 \mathrm{~cm}$ on imaging. On resection, true size was $10 \times 5 \mathrm{~cm}$.

lapse.

Reviewing her postoperative diagnostic workup also highlights the value of transthoracic echocardiography as a quick, non-invasive tool in the evaluation of postoperative respiratory distress. The most likely order of common causes of acute respiratory distress following noncardiac surgery includes pulmonary embolism, pneumonia, pneumothorax, atelectasis-induced hypoxemia and heart failure. Diagnostic imaging tools like CT scan and magnetic resonance imaging (MRI) require moving a potentially unstable patient, and at times administering contrast. In the described case, a bedside echocardiogram would have immediately shown the underlying cause of the patient's distress, and the need for surgical intervention would have been established.

In conclusion, we suggest left atrial myxoma be in- cluded in the differential diagnosis of acute postoperative respiratory distress following non-cardiac surgery. We propose that echocardiography should be the first choice of imaging in these conditions.

\section{REFERENCES}

[1] G. Ferreyra, Y. Long and V. Ranieri, "Respiratory Complications after Major Surgery," Current Opinion in Critical Care, Vol. 15, No. 4, 2009, pp. 342-348. doi:10.1097/MCC.0b013e32832e0669

[2] C. Owers, P. Vaughan, P. Braidley, G. Wilkinson, T. Locke, G. Cooper, N. Briffa, D. Hopkinson and P. Sarkar, "Atrial Myxomas: A Single Unit's Experience in the Modern Era,” Heart Surgery Forum, Vol. 14, No. 2, 2011, pp. 105-109. doi:10.1532/HSF98.20091163

[3] J. Butany, V. Nair, A. Naseemuddin, G. Nair, C. Catton, and T. Yau, "Cardiac Tumours: Diagnosis and Management," Lancet Oncology, Vol. 6, No. 4, 2005, pp. 219228. doi:10.1016/S1470-2045(05)70093-0

[4] M. Panagiotou, N. Panagoulos, P. Ravazoula, L. Kaklamanis and E. Koletsis, "Large Asymptomatic Atrial Myxoma with Ossification: Case Report,” Journal of Cardiothoracic Surgery, Vol. 29, No. 3, 2008, pp. 19-21. doi:10.1186/1749-8090-3-19

[5] J. Walpot, B. Shivalkar, I. Rodrigus, W. H. Pasteuning and R. Hokken, "Atrial Myxomas Grow Faster than We Think,” Echocardiography, Vol. 27, No. 10, 2010, pp. E128-E131. doi:10.1111/j.1540-8175.2010.01186.x

[6] T. Matsushita, A. Huyn, T. Singh, P. Hayes, S. Armarego and P. Sea, "Mitral Valve Annular Dilatation Caused by a Left Atrial Myxoma," Heart, Lung and Circulation, Vol. 18, No. 2, 2009, pp. 145-147. doi:10.1016/j.hlc.2007.10.017 